\title{
Free Versus Pedicled Anterolateral Thigh Flap for Abdominal Wall Reconstruction
}

\author{
PIETRO G. DI SUMMA ${ }^{1 *}$, WILLIAM WATFA ${ }^{1 *}$, CORRADO CAMPISI $^{2}$, \\ SALVATORE GIORDANO ${ }^{3}$, CARLO M. ORANGES $^{4}$, LESLIE ELAHI-RAUSIS $^{1}$, \\ OLIVIER BAUQUIS ${ }^{1}$, DIETER HAHNLOSER ${ }^{5}$, NICOLAS DEMARTINES ${ }^{5}$ and WASSIM RAFFOUL ${ }^{1}$ \\ ${ }^{1}$ Department of Plastic, Reconstructive and Hand Surgery, \\ University Hospital of Lausanne (CHUV), Lausanne, Switzerland; \\ ${ }^{2}$ Department of Surgery, Unit of Lymphatic Surgery, S. Martino Hospital, University of Genoa, Genoa, Italy; \\ ${ }^{3}$ Department of Plastic and Reconstructive Surgery, Turku University Hospital, Turku, Finland; \\ ${ }^{4}$ Department of Plastic, Reconstructive and Hand Surgery, University Hospital of Basel, Basel, Switzerland; \\ ${ }^{5}$ Department of Visceral Surgery, University Hospital of Lausanne (CHUV), Lausanne, Switzerland
}

\begin{abstract}
Background/Aim: Large full thickness abdominal wall defects following malignancies can be a reconstructive challenge. The purpose of this study was to analyze longterm outcomes and complications following abdominal wall reconstruction using composite antero-lateral thigh (ALT) flaps. Patients and Methods: The study retrospectively investigated 16 consecutive patients who underwent abdominal wall reconstruction with autologous flap between May 2003 and March 2018. Volumetric flap analysis was used to assess flap atrophy over time, evaluating the role of denervation and reinnervation. The long-term outcome was assessed to compare the two groups (free vs. pedicled ALT flap reconstructions). Results: All flaps successfully covered the defects. We found a significant increase in flap resorption in free flaps when compared to pedicled ones. Abdominal bulging was seen in 3 out of 16 (19\%) patients after more than 12 months follow-up, in close correlation with mesh absence. Conclusion: Free flaps were shown to be equally effective as their pedicled counterparts, without significant increase in complication rate.
\end{abstract}

This article is freely accessible online.

*These Authors have equally contributed to this study.

Correspondence to: PD\&MER Dr. Pietro di Summa, MD, PhD, FEBOPRAS, FMH (Plast.), Consultant, Department of Plastic, Reconstructive and Aesthetic Surgery, Lausanne University Hospital, Lausanne, Switzerland. Tel: +41 0213142525, Fax: +41 0213142530, e-mail: pietro.di-summa@chuv.ch

Key Words: Abdominal wall reconstruction, abdominal wall stability, composite anterolateral thigh flap, vascularized fascia lata.
Abdominal wall defects remain a challenge for reconstructive surgeons. They can range from only soft tissue defects resulting from peritonitis and laparotomies (1-4), to extensive threelayered (soft tissue, muscle and abdominal fascia) full thickness defects following en bloc resection of tumors (5). The ideal management of abdominal wall reconstruction (AWR) should aim to re-establish the three essential structures in order to assure a stable soft-tissue coverage, a solid fascial component to protect the viscera and minimize infection and hernia recurrence, and a muscular component possibly aiming for a functional return (6, 7). Among described surgical treatments, component release surgery remains popular (8), along with flap surgery and the fast growing advances in acellular dermal matrices (ADM).

In case of recurrent eventration without enterocutaneous fistulas, surgical mesh closure associated with component release surgery is often the first-line intervention, considering the affordable price, the ease and speed of the procedure (9). Litterature shows that synthetic nondegradable or degradable meshes are not recommended for infected fields, where biological meshes are more suitable $(7,10-12)$. However, ADMs are more expensive and might be associated with higher hernia recurrence rate $(13,14)$.

In most cases of full thickness defects including skin $(e . g$. after tumour resection) or when meshes are infected, flaps are preferentially used (15). Large abdominal wall defect reconstructions represent critical challenges because of the complexity of the anatomical structures to reconstruct, and of the scarred, infected or radiated environment where the flap is transferred. Furthermore, they often require a bridged mesh repair because of difficulties in fascial approximation, increasing hernia recurrence risk $(16,17)$.

Many pedicled flaps have been described in AWR, such as external obliques (18), rectus abdominis (19) and the antero-lateral thigh (ALT) myocutaneous flap (20). Despite 
being less frequent, free tissue transfer has been described, using the latissimus dorsi (3), rectus femoris (21), tensor fascia lata and the free ALT myocutaneous flap, which remains the preffered option due to its anatomical properties and versatility (22). Indeed, the composite ALT flap (including vastus lateralis and fascia lata) holds interesting features in AWR. The fascia lata adds a solid component that could potentially decrease the risk of eventration, while the vastus lateralis could mimic the dynamic function of the rectus abdominis muscle when reinnervated $(20,23,24)$.

However, the long-term performance and functional outcomes of composite ALT flaps in AWR are still unknown, together with the role of muscle atrophy and loss of fascia tensile strength over time. Moreover, previous studies have analyzed the differences between the use of free flap over pedicled flap as structural support in AWR $(22,25)$, however, few have specifically focused on the use of ALT. Also, no reports have analysed the potential differences due to flap denervation/reinnervation when a free tissue transfer is performed.

In this study, ALT flaps were tailored depending on the location and features of the defect and, transferred as pedicled or innervated free flaps for AWR. All reconstructions were compared taking into consideration immediate outcomes and long-term functional results. In order to investigate the relation between flap integrity and clinical outcomes, volumetric imaging analysis was performed at different timepoints. This work analyzed longterm outcomes and complications following abdominal wall reconstruction using composite ALT flaps, either pedicled or free, and ideally guided according to defect localisation, properties, and eventual mesh position.

\section{Patients and Methods}

Patient data. Between May 2003 and March 2018, 16 patients presenting complex abdominal wall defects were admitted to our Department for reconstruction (all involving fascia, muscles and generally skin with subcutaneous tissues). The two main etiologies were invasive neoplasias and recurrent eventration following visceral surgery complications (Kanters Modified Hernia Grade 2 and 3A) (26). The mean age of the 16 patients ( 9 males, 7 females) was $58 \pm 3$ years (average \pm SEM). For all patients, past medical history was retrieved from their hospital medical records, which included metabolic syndrome (5/16), smoking (4/14), previous multiple abdominal interventions (12/16); average number of interventions: 3.8 , previous neoplastic diseases $(8 / 16)$. If an infected mesh was present, removal and replacement with a new one was performed by the team of visceral surgeons at the same time of the flap coverage (Table I). Written consent was obtained from all patients, and the procedures were performed in line with the Helsinki Declaration.

Surgical technique. After suitable design of the flap according to the requirements of the defect, incision was made in the medial line of the skin paddle and deepened down to the muscular aponeurosis. The descending branch of the lateral circumflex femoral artery (LCFA) was identified in the intermuscular septum between the rectus femoris and the vastus lateralis. Mapped perforators, generally two, were identified and the skin paddle was designed accordingly. All ALT flaps were harvested together with vastus lateralis and a segment of fascia lata as composite flaps.

In most cases, we performed anterior component release and placed a surgical mesh before covering the defect with a flap. When the meshes were placed in a bridging technique, a larger fascia lata segment was harvested together with the ALT-VL flap, to reinforce the meshes. When a pedicled transposition of the flap was planned (11 out of 16 flaps, $69 \%$ ), the LCFA was generally followed proximally at its origin or up to the deep femoral vessels and the flap was transposed passing under the Sartorius or rectus femoris muscle (Figure 1). This tunnelled flap allowed us to reach defects over the umbilicus avoiding tension or twisting of the pedicle. Only one patient (patient 8 ) required pedicle nerve division, to allow complete cranial mobilisation and tension-free pedicle positioning.

When a free tissue transfer was required (5 out of 16 flaps, $31 \%$ ), the descending branch of the LCFA was generally followed until the rectus branch, where the calibre of vessels matched the size of receiving vessels (deep inferior epigastric artery and vein, DIEA/V). Anastomoses to DIEA/V were performed end-to-end with 8-0 nylon sutures under a microscope. All free flaps except one (patient 11) were reinnervated using the biggest motor branch available (generally a lower branch) from the remnant rectus abdomini muscles, and coapted to the femoral nerve branch directed to the vastus lateralis muscle. Similarly, nerve coaptation was performed end-to-end with a 8-0 nylon under a microscope, followed by the application of Tisseel Glue (Baxter, Baxter International Inc., Deerfield, IL, USA) around the nerve coaptation site (Figures 1 and 2). Intravenous antibiotic therapy was given preoperatively and continued according to microbiological findings.

Functional outcomes and hernia recurrences. Patient's functional assessment included physical exam for possible abdominal herniation, and quality of life assessment at 1-year follow-up, when The HerniaRelated Quality-of-life Survey (HerQLes) questionnaire was submitted to the patients. This is a validated questionnaire in order to evaluate the quality of life as it relates to the abdominal wall function (27).

Radiological flap volume assessment. Radiological follow-up [computed tomography (CT), GE Medical Systems Lightspeed VCT, General Electric Corporate, Fairfield, CT, USA; magnetic resonanse imaging (MRI), Magnetom Siemens Skyra 3T, Siemens Healthcare, Munich, Germany] was performed only on 9 out of 16 (56\%) patients who had a defect following tumor extirpation due to the risk of oncological recurrence and in order to avoid unnecessary radiation. Indeed, in patients who had reconstructions following infectious etiologies, trauma or general surgery complications without oncological history, follow-up CTs were performed only under clinical suspicion of infection recurrences or presence of fistulas. Images at different timepoints were useful to precisely evaluate flap efficiency in terms of dead space coverage, and to quantitatively evaluate percentage global bulk atrophy over time. Flap volumes were performed at 3 and 6 months and every year postoperatively. Volumetric analysis was performed on CT scans or MRI images (Carestream Vue PACS, Carestream Health, Rochester, NY, USA) (Figure 3). Mean follow-up was 30.1 \pm 3.4 months (average \pm SEM). In each cross section (ranging from 2 to $5 \mathrm{~mm}$ thickness), different flap surfaces from caudal to cranial were assessed. The volume of every section (surface section thickness) was obtained; then the sum of the 
different section volumes yielded the total flap volume (TFV), as previously described (28). Measurements were performed by an external examiner, blind to both the study and the different patients. Specifically for volumetric analysis, patients were divided into 3 groups: nerve-preserved flaps, denervated flaps and re-innervated free flaps. TFVs at different timepoints were compared to the initial flap volume (3 months postoperatively), and percentage differences in volume resorption were compared among groups.

Donor site assessment. Based on physical exam, no patient described limitations at the donor site although two patients (patients 7 and 8) required split thickness skin graft (STSG) at the donor site. Indeed, ALT harvesting, even if it included vastus lateralis muscle, did not influence range of motion (ROM) at the knee level in terms of flexion-extension, and all retained M5 force compared with the contralateral side.

Statistical analysis. All investigated parameters (general data, functional) were statistically analysed (average, range, standard error of the mean) with GraphPad Prism 6.00 (GraphPad Software, La Jolla, CA, USA). Unpaired Student $t$-test was used to determine any significant difference between pedicled and free flaps regardless of their innervation status, when investigating usually measured parameters such as operative time, time to healing (when stitches were generally removed), hospital stay and defect size. On the other hand, one-way ANOVA multiple comparison test was used to evaluate differences in flap volume between nerve-preserved flaps, denervated flaps and reinnervated free flaps. Significance was determined as $* p<0.05, * * p<0.01$.

\section{Results}

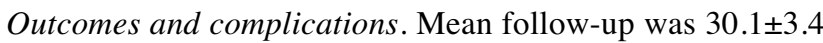
months (average \pm SEM). At the time of referral, all 16 patients presented with previous multiple abdominal interventions, and all reconstructions were performed with ALT composite flaps. The average fascial defect after the oncologic procedure was $121.6 \pm 20.46 \mathrm{~cm}^{2}$ (average \pm SEM) for pedicled flaps and $162 \pm 35.97 \mathrm{~cm}^{2}$ (average \pm SEM) for free flaps. Regarding the cutaneous defect, pedicled flaps average was $229.3 \pm 22.54 \mathrm{~cm}^{2}$ (average \pm SEM) and $160.5 \pm 18.08 \mathrm{~cm}^{2}$ (average \pm SEM) for free flaps (Table I).

All flaps included part of the vastus lateralis muscle and fascia lata to rebuild abdominal fascia loss of substance. Eleven patients (69\%) who had a defect located in the infraumbilical \pm periumbilical zone were covered by pedicled flaps, while five patients $(31 \%)$ presented a defect involving both lower and upper abdomen and benifited from coverage with a free flap. No statistically significant difference in terms of fascia and skin size defect was present between pedicled and free flaps (Figure 4). The average reconstruction operative time was $326.3 \pm 31.73 \mathrm{~min}$ (average \pm SEM) for pedicled flaps and $409 \pm 30.01 \mathrm{~min}$ (average \pm SEM) for free flaps. Despite a slight trend for longer operative time in free flaps, no statistical significant difference was present (nss; $p=0.13$ ).

Average time to healing was $20.27 \pm 1.54$ days (average \pm SEM) for pedicled flaps and 18.8 \pm 2.6 days (average \pm SEM) for free flaps. Average hospitalization time was $23.1 \pm 4.13$ days (average \pm SEM) for pedicled flaps and $20.20 \pm 1.83$ days (average \pm SEM) for free flaps. Analysis showed no statistical difference between these groups.

Three patients presented minor dehiscence at the flap inset site, which healed uneventfully with conservative measures in less than 6 weeks. Patient 6 developed a postoperative hemato-seroma under full dose anticoagulation, requiring surgical drainage. Patient 12 presented an early postoperative collection under the flap, which required washout and intravenous antibiotic treatment. All patients healed uneventfully with no flap-related complications.

Functional outcomes and abdominal wall complications. Three patients (patients $4,11 \& 12$ ) developed postoperative bulging (19\%). None of these cases had benefited from the immediate use of meshes but relied solely on the composite flap coverage. Patient 4 who presented with a late abdominal bulge (40 months postoperative) following a pedicled ALT reconstruction did not report any interference with her daily activities and therefore refused any further interventions. Patient 11 , who had a free flap reconstruction without reinnervation, required a secondary positioning of a mesh to contain the abdominal content in the lower right abdominal quadrant. Patient 12 benefited from a free reinnervated flap, but presented abdominal bulge in valsalva at 2 years follow-up (Figure 5). Despite aesthetic concerns, no functional abdominal-related symptoms were present in this last case and did not require re-intervention.

The average HerQLes score reflecting quality of life after abdominal wall reconstruction at 12 months postoperative was $26.18 \pm 2.46$ (average \pm SEM) for pedicled group and $30.8 \pm 3.17$ (average \pm SEM) for free flap group. No statistically significant difference was present.

Radiological follow-up and flap volumetric quantitative analysis. No signs of dead space, partial or total flap necrosis were noticed during imaging follow-up. Moreover, flap pedicles were still visible in angioCTs, confirming vessel patency and efficient flap vascularisation (Figure 3). Volumetric analysis data from the 8 patients who underwent serial radiological studies, were statistically analysed (3 nervepreserved, 3 reinnervated free flaps, 2 denervated). The average flap volume (average of volumes at different time points) of each patient was calculated, ranging from $184 \mathrm{~cm}^{3}$ (patient 2) to $820 \mathrm{~cm}^{3}$ (patient 6) with a global average of $466 \pm 152 \mathrm{~cm}^{3}$ (average \pm SEM). We found a significant increase in the resorption percentage of volume over 12 months in free flaps when compared to pedicled ones $\left({ }^{* *} 9.67 \pm 0.88\right.$ in pedicled group vs. $22 \pm 1$ in free transfer group; values expressed as average \pm SEM). The patient on whom flap was denervated (patient 5) was not included into the group analysis. However, CT flap resorption was measured, accounting for an even higher flap resorption (31\% at 12 months). 


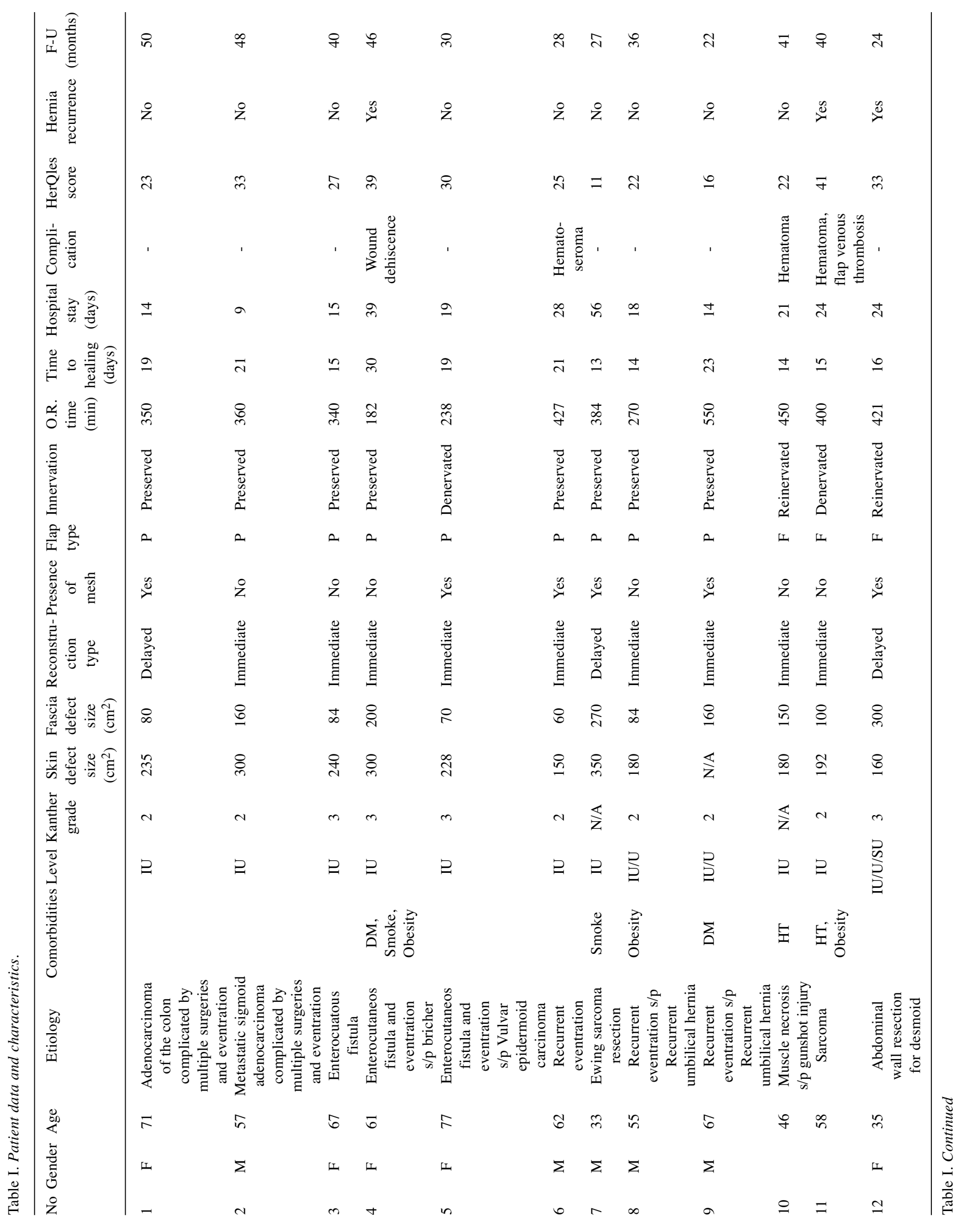




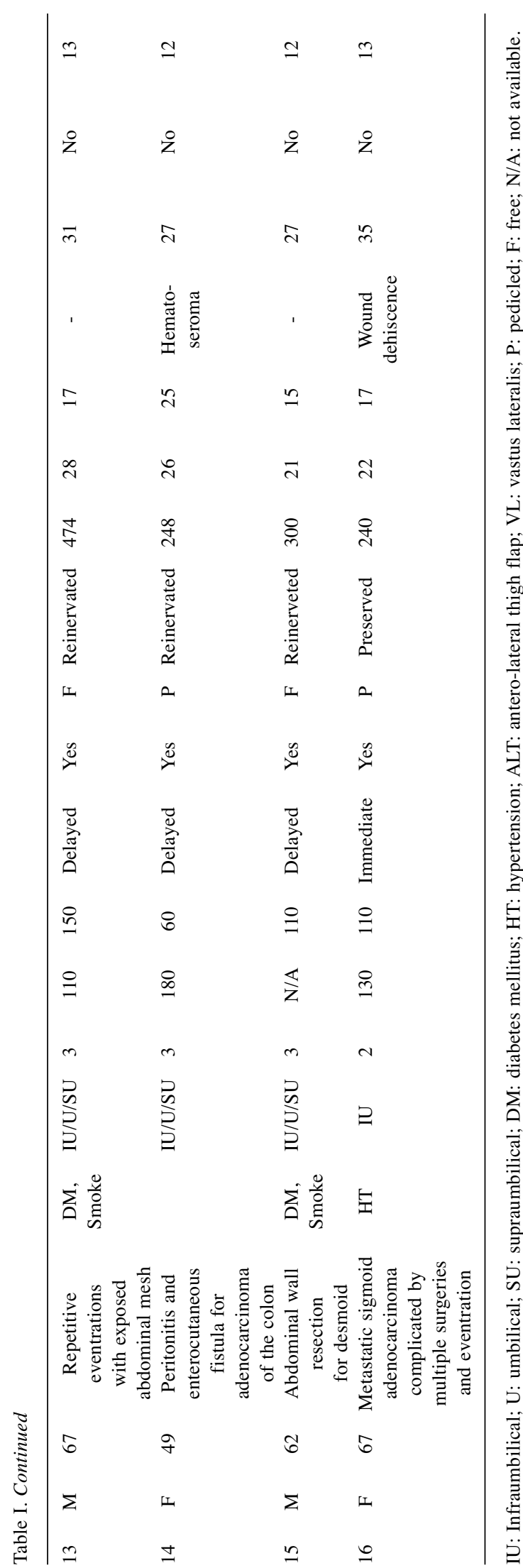
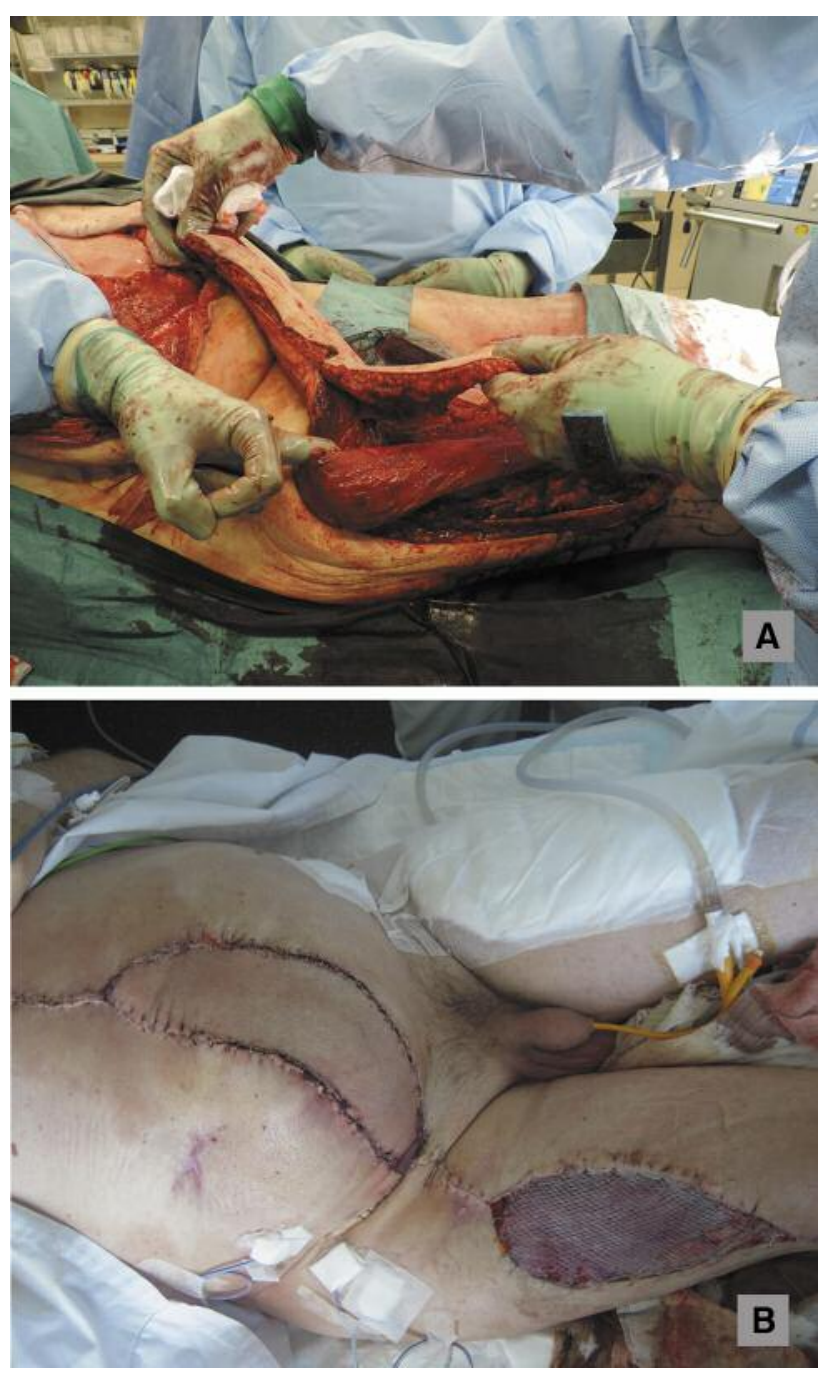

Figure 1. Pedicled ALT transposition: for further cranial reach, the flap was transposed passing under the sartorius or rectus femoris (A). Early postoperative result in patient 8 who required a split-thickness skin graft for sonnor site closure $(B)$.

\section{Discussion}

Composite abdominal wall defects are particularly complex to reconstruct, especially when bowel eventration, enterocutaneous fistulas and mesh contaminations challenge the reconstructive aim of restoring transmural continuity, and possibly re-establishing the structural and functional properties of the abdominal wall (15). In 2000, Mathes et al. showed that flap surgery had to be combined with complex abdominal reconstruction especially after previous failed attempts with infected mesh (4). Indeed, in hostile milieu, flaps can bring healthy vascularized tissue to the meshed area, offering a better antibiotic delivery and wound healing, in addition to obliterating the area $(20,29)$. 

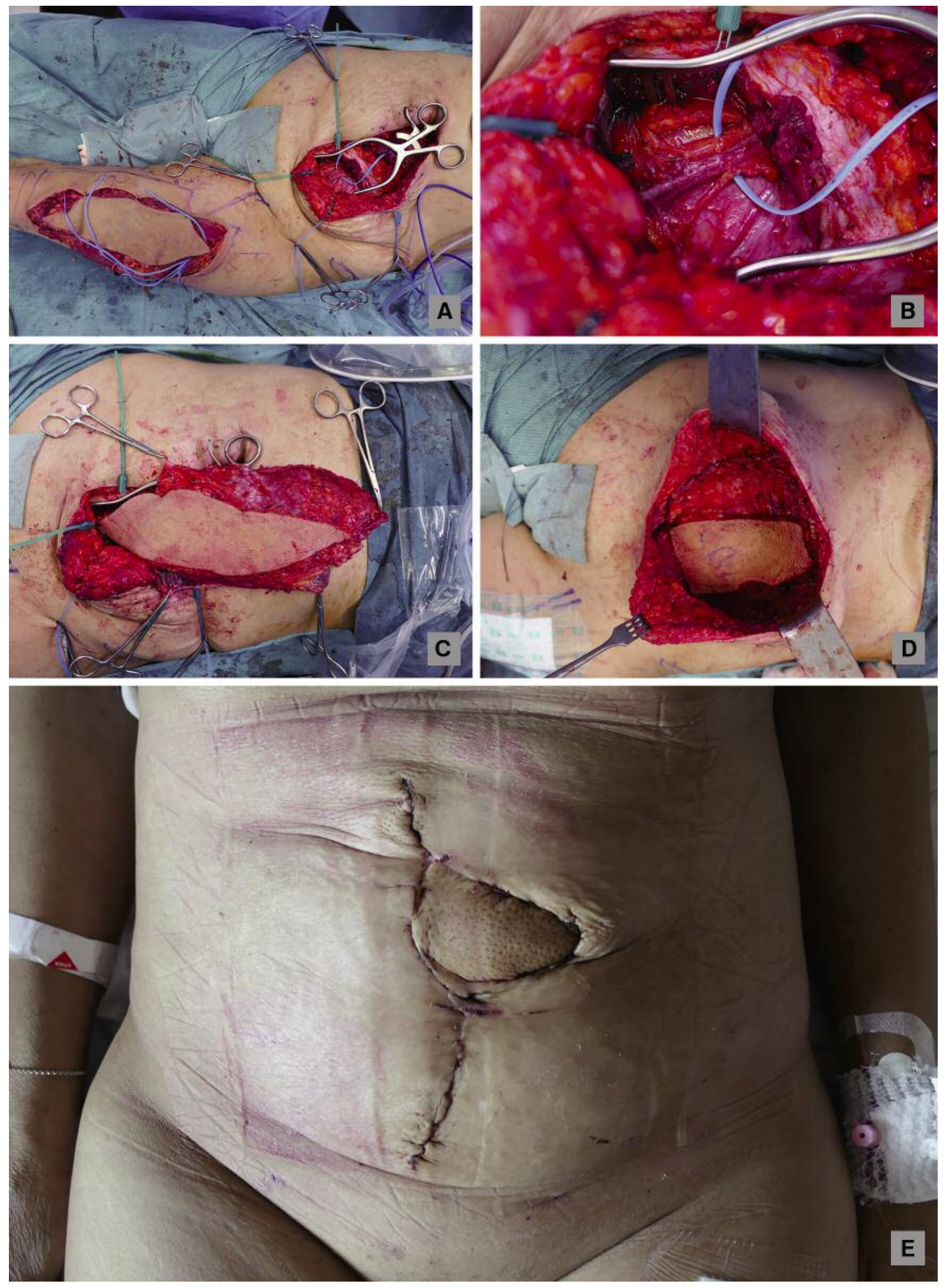

Figure 2. Intraoperative steps of the free flap with coverage of the fascial defect (Patient 14). The composite ALT flap includes fascia lata and the vastus lateralis muscle allowing reinforced support while replacing the missing components of the abdominal wall musculature. (A) Free ALT flap raised and ready to be transferred. (B) End to end anastomoses on the deep inferior epigastric vessels, together with nerve coaptation to a motor branch of the rectus abdominis muscle. (C) Revascularised flap showing fascia lata and muscular components. (D) Reconstructed fascial wall. (E) Postoperative result with monitor skin paddle. 

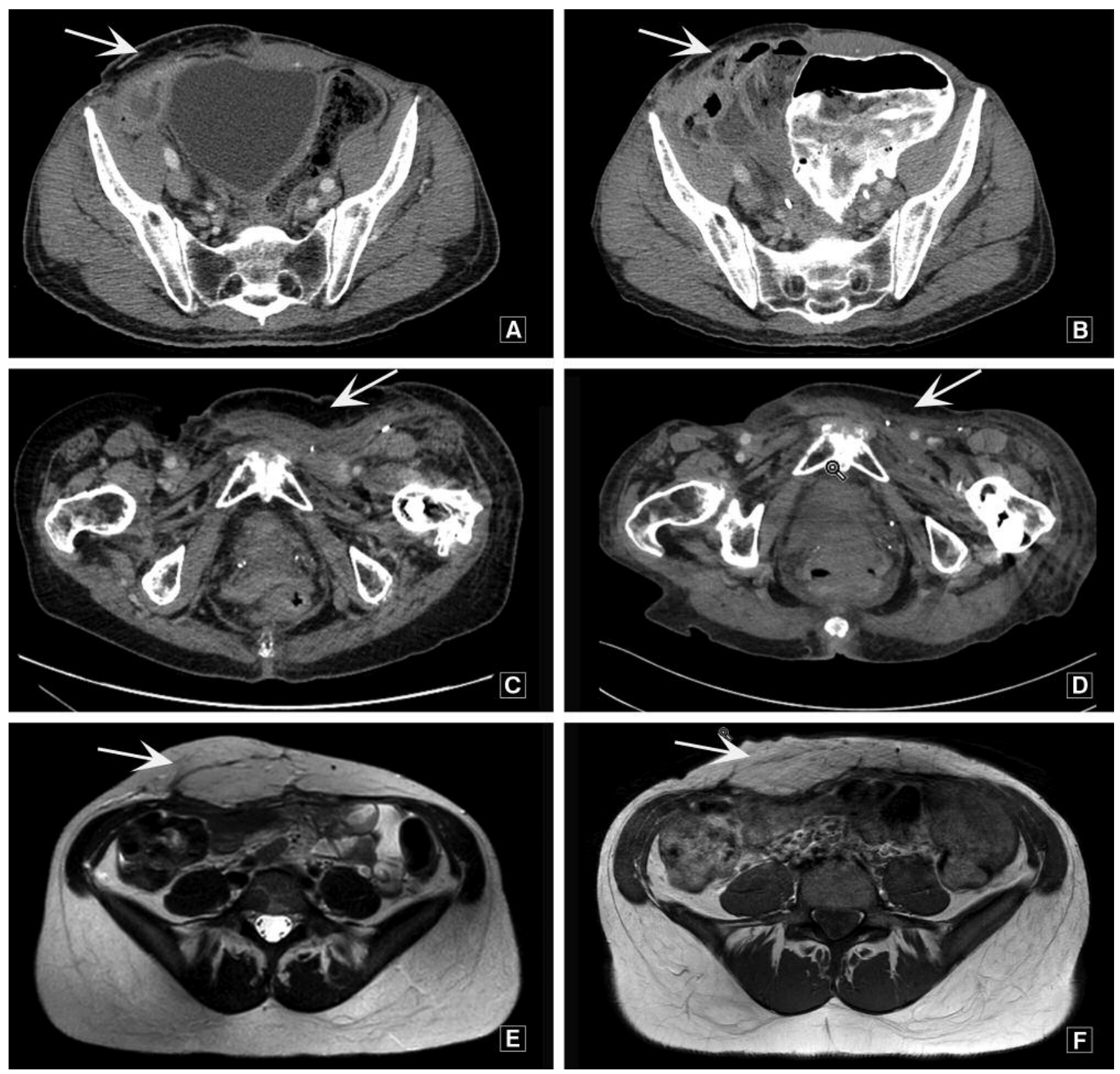

Figure 3. Serial imaging showing the ALT flap (white arrow) resorption over time. Serial CT scans at follow-up of patient 2 (preserved innervation) after pedicled ALT reconstruction for a 3-dimensional abdominal defect. Six months postoperative (A). 12 months postoperative (B). Serial CT scans at follow-up of patient 5 (denervated) after pedicled ALT reconstruction for a 3-dimensional abdominal defect. Six months postoperative (C). At 12 months postoperative $(D)$. Serial MRI at follow-up of patient 10 (reinnervated) after free ALT reconstruction for a 3-dimensional abdominal defect. Six months postoperative (E). At 12 months postoperative $(F)$.

The ALT is the workhorse for soft-tissue reconstruction and has been successfully used for abdominal wall defects $(22,25,30,31)$. Vranckx et al. presented in 2015 the PIVA flap concept consisting of a pedicled ALT with an innervated vastus lateralis (20). In their series of 16 patients, dynamic properties of the flap were measured by isokinetic measurements during flexion/extension of the abdominal wall, electromyography (EMG) and a validated quality-of-life questionnaire. Their results at one year showed that the abdominal wall had developed into a dynamically controllable unit. EMG performed on the vastus lateralis showed good contractility, which goes along with our belief of restoring dynamic structure with the use of composite flaps. 


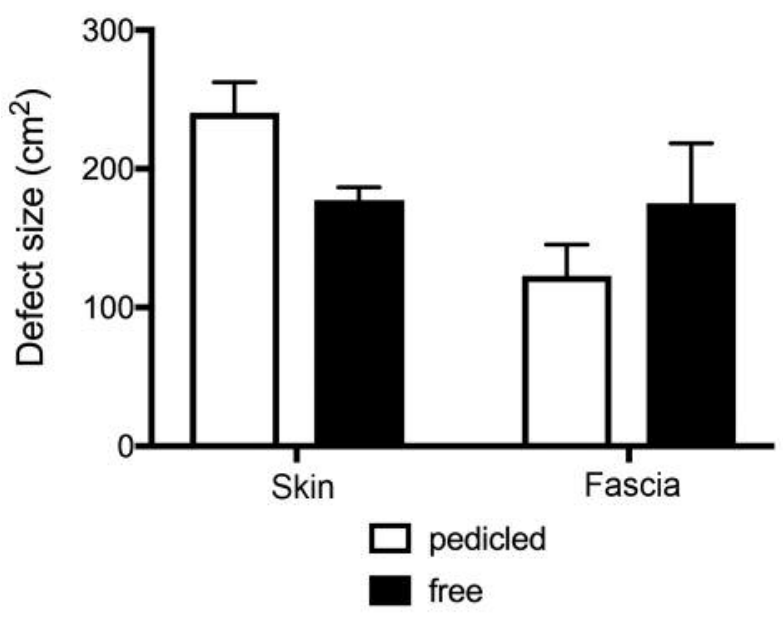

Figure 4. Average surface in $\mathrm{cm}^{2}$ for fascial and cutaneous defects in pedicled $v$ s. free flap reconstruction.

As recently reviewed, reconstruction of the muscular components of the abdomen should be considered, especially when the defect includes more than rectus abdominis muscles (32). However, the behaviour over time of innervated (pedicled) or re-innervated (33) flaps, and the respective roles of fascia/muscle components in the stabilisation of the abdominal unit, has yet to be fully investigated.

No difference in flap related complication rates between pedicled and free ALT was noticed, aside from a slightly longer operative duration related to the microsurgical time, which did not reach a statistically significant difference. Similarly, time to complete healing and length of hospital stay were similar between the two groups.

Volumetric measurements and imaging confirmed flap stability over time, assuring coverage, support and avoiding dead space (Figure 3). Data on flap volume decrease provided us with essential information to support our idea that abdominal flaps can sufficiently resist atrophy, potentially maintaining a dynamic function (20). As expected, free re-innervated flaps showed a significant volume loss when compared to their pedicled innervated counterpart. However, resorption was limited to $20 \%$ of volume loss, comparing favourably to the denervated flaps, which showed a resorption consistently over $30 \%$. Indeed, in our series, stability of reconstruction seemed to be more influenced by the reconstitution of an effective fascia layer associated with meshes, than by muscle bulk (Figure 5). This was particularly evident in those patients where no synthetic meshes were inserted (for high infective risk, or because of clinical judgement), relying solely on the free ALT-VL-FL. Despite excellent flap outcomes in terms of coverage and reestablishment of anatomical layers, the long-term outcome
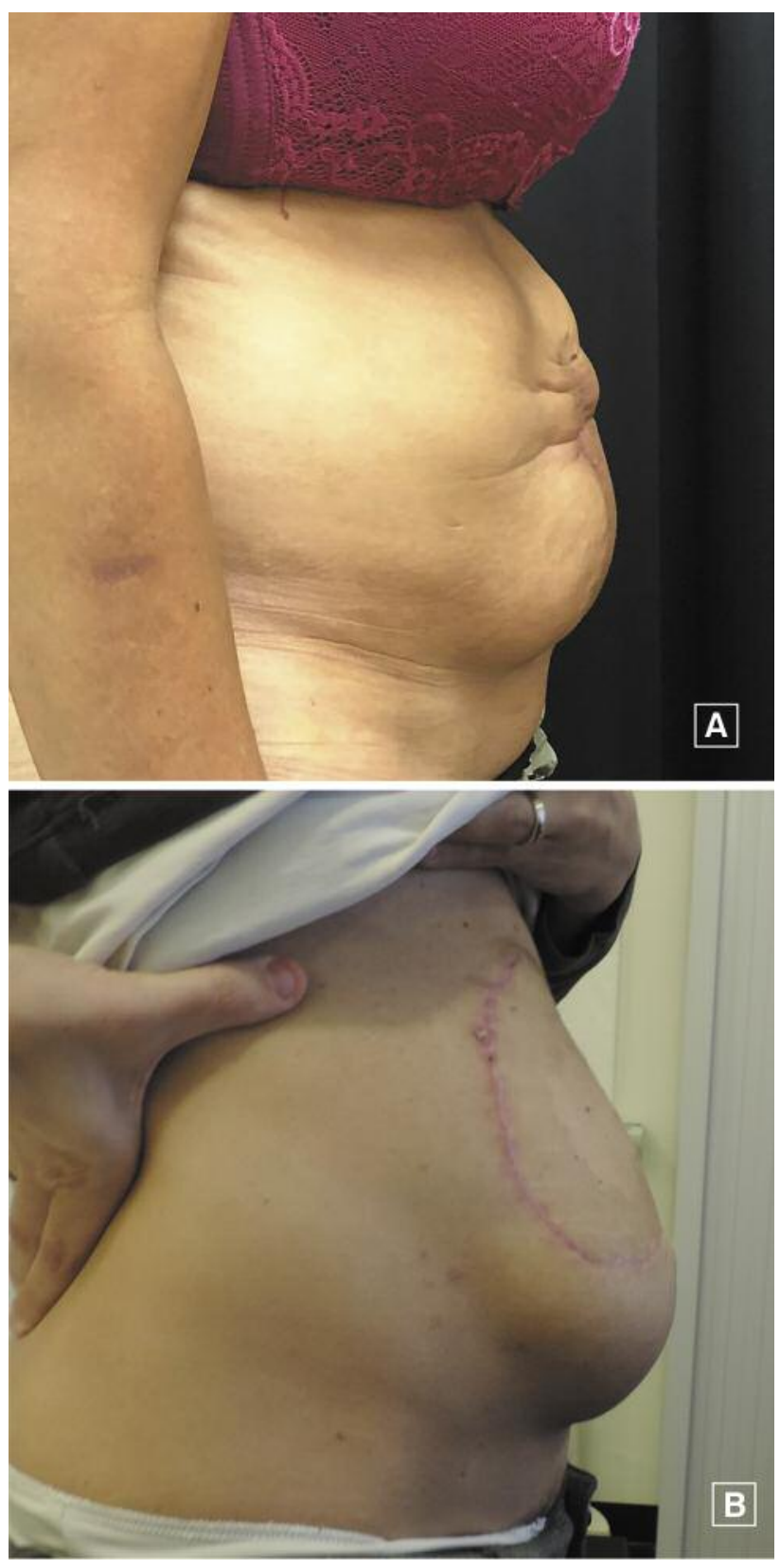

Figure 5. One-year postoperative lateral view of AWR during valsalva maneuver. Patient 14 (free flap with mesh shown in Figure 2) showing good abdominal wall integrity (A). Patient 12 (free flap without mesh) showing abdominal bulge $(B)$.

of these reconstructions shows that even a resistant tissue component like FL undergoes progressive stretch under intrabdominal continuous pressure. The almost total absence of rectus abdominis muscle to contrast intrabdominal pressure had probably a further role in bulge recurrence. Abdominal wall functional questionnaires (HerQLes) on 
quality of life did not show any differences regardless of the type of reconstruction, and only one patient required a secondary mesh placement.

Defect location, rather than defect size (Figure 4), influenced the reconstructive choice as pedicled transposition to the upper abdominal quadrant is limited by the thigh length/trunk ratio (20). ALTs in the pedicled form were mostly used when the location of the defect was in the central or lower abdomen $(20,34)$ : transposition to reach the upper quadrants may be tedious (20), and requires an extensive pedicle dissection up to the profunda artery, occasionally requiring nerve division (patient 5). When defects were multiple, or extended caudo-cranially, free flaps allowed for better insetting and were generally preferred.

Despite the severity of the defects in this series, hernia recurrence rate compares favorably with the literature (16, 35). Flap atrophy did not seem to have significant repercussions on abdominal wall stability, at least from a clinical point of view. This goes in line with the concept that fascia layers retain a more determinant role than muscle integrity in preserving abdominal wall stability, as suggested by recent studies comparing donor site morbidity after muscle-sparing transverse rectus abdominis myocutaneous (MS-TRAM) vs. deep inferior epigastric perforator (DIEP) flaps, showing similar hernia rates $(36,37)$.

This study showed how AWR with composite ALT flaps can provide vascularized fascia layers, which can reinforce prosthetic meshes, reducing hernia recurrences and abdominal wall infections. However, the retrospective investigation (even if conducted on a prospectivelymaintained database), and the relatively small number of patients included, need to be underlined.

\section{Conclusion}

ALT composite flaps can efficiently reconstruct the abdominal wall, without significant difference in outcomes between free and pedicle forms. Mimicking the original abdominal components, missing after oncological resection, this flap guarantees a structured reconstruction and an efficient coverage of all abdominal wall areas.

\section{Conflicts of Interest}

The Authors declared no potential conflicts of interest with respect to the research, authorship, and publication of this article.

\section{Authors' Contributions}

William Watfa performed the literature review and drafted the manuscript. Leslie Elahi-Rausis performed the aquisition of data. Pietro G. di Summa conceived the topic of the article, supervised the study and drafted revisions. Wassim Raffoul, Corrado Campisi, Salvatore Giordano, Carlo M. Oranges, Olivier Bauquis, Dieter Hahnloser and Nicolas Demartines proof-read and critiqued the manuscript. All Authors read and approved the final manuscript.

\section{Acknowledgements}

The Authors received no financial support for the research, authorship, and publication of this article.

\section{References}

1 Gottlieb JR, Engrav LH, Walkinshaw MD, Eddy AC and Herman CM: Upper abdominal wall defects: Immediate or staged reconstruction? Plast Reconstr Surg 86(2): 281-286, 1990. PMID: 2142309. DOI: 10.1097/00006534-199008000-00013

2 DeFranzo AJ, Kingman GJ, Sterchi JM, Marks MW and Thorne MT: Rectus turnover flaps for the reconstruction of large midline abdominal wall defects. Ann Plast Surg 37(1): 18-23, 1996. PMID: 8826587. DOI: 10.1097/00000637-199607000-00003

3 Ninkovic M, Kronberger P, Harpf C, Rumer A and Anderl H: Free innervated latissimus dorsi muscle flap for reconstruction of full-thickness abdominal wall defects. Plast Reconstr Surg 101(4): 971-978, 1998. PMID: 9514329. DOI: 10.1097/00006 534-199804040-00013

4 Mathes SJ, Steinwald PM, Foster RD, Hoffman WY and Anthony JP: Complex abdominal wall reconstruction: A comparison of flap and mesh closure. Ann Surg 232(4): 586-596, 2000. PMID: 10998657. DOI: 10.1097/00000658-200010000-00014

5 Khansa I and Janis JE: Modern reconstructive techniques for abdominal wall defects after oncologic resection. J Surg Oncol 111(5): 587-598, 2015. PMID: 25371050. DOI: 10.1002/jso.23824

6 Butler CE, Baumann DP, Janis JE and Rosen MJ: Abdominal wall reconstruction. Curr Probl Surg 50(12): 557-586, 2013. PMID: 24231005. DOI: 10.1067/j.cpsurg.2013.08.003

7 Ghazi B, Deigni O, Yezhelyev M and Losken A: Current options in the management of complex abdominal wall defects. Ann Plast Surg 66(5): 488-492, 2011. PMID: 21372667. DOI: 10.1097/SAP.0b013e31820d18db

8 Ramirez OM, Ruas E and Dellon AL: "Components separation" method for closure of abdominal-wall defects: An anatomic and clinical study. Plast Reconstr Surg 86(3): 519-526, 1990. PMID: 2143588. DOI: 10.1097/00006534-199009000-00023

9 Denney B and de la Torre JI: Multipoint suture fixation technique for abdominal wall reconstruction with component separation and onlay biological mesh placement. Am Surg 83(5): 515-521, 2017. PMID: 28541865.

10 Rastegarpour A, Cheung M, Vardhan M, Ibrahim MM, Butler $\mathrm{CE}$ and Levinson $\mathrm{H}$ : Surgical mesh for ventral incisional hernia repairs: Understanding mesh design. Plast Surg (Oakv) 24(1): 41-50, 2016. PMID: 27054138.

11 Diaz JJ Jr., Conquest AM, Ferzoco SJ, Vargo D, Miller P, Wu YC and Donahue R: Multi-institutional experience using human acellular dermal matrix for ventral hernia repair in a compromised surgical field. Arch Surg 144(3): 209-215, 2009. PMID: 19289658. DOI: 10.1001/archsurg.2009.12

12 Garvey PB, Giordano SA, Baumann DP, Liu J and Butler CE: Long-term outcomes after abdominal wall reconstruction with acellular dermal matrix. J Am Coll Surg 224(3): 341-350, 2017. PMID: 27993696. DOI: 10.1016/j.jamcollsurg.2016.11.017 
13 Hubner M, Streit D and Hahnloser D: Biological materials in colorectal surgery: Current applications and potential for the future. Colorectal Dis 14: 34-39, 2012. PMID: 23136823. DOI: 10.1111/codi. 12048

14 Janis JE, O'Neill AC, Ahmad J, Zhong T and Hofer SO: Acellular dermal matrices in abdominal wall reconstruction: A systematic review of the current evidence. Plast Reconstr Surg 130: 183S-193S, 2012. PMID: 23096969. DOI: 10.1097/ PRS.0b013e3182605cfc

15 Ko JH, Wang EC, Salvay DM, Paul BC and Dumanian GA: Abdominal wall reconstruction: Lessons learned from 200 "components separation" procedures. Arch Surg 144(11): 10471055, 2009. PMID: 19917942. DOI: 10.1001/archsurg.2009.192

16 Giordano S, Garvey PB, Baumann DP, Liu J and Butler CE: Primary fascial closure with biologic mesh reinforcement results in lesser complication and recurrence rates than bridged biologic mesh repair for abdominal wall reconstruction: A propensity score analysis. Surgery 161(2): 499-508, 2017. PMID: 27810091. DOI: $10.1016 /$ j.surg.2016.08.009

17 Sosin M, Nahabedian MY and Bhanot P: The perfect plane: A systematic review of mesh location and outcomes, update 2018. Plast Reconstr Surg 142: 107S-116S, 2018. PMID: 30138278. DOI: $10.1097 / P R S .0000000000004864$

18 Althubaiti G and Butler CE: Abdominal wall and chest wall reconstruction. Plast Reconstr Surg 133(5): 688e-701e, 2014. PMID: 24776572. DOI: 10.1097/PRS.0000000000000086

19 Kushimoto S, Yamamoto Y, Aiboshi J, Ogawa F, Koido Y, Yoshida R and Kawai M: Usefulness of the bilateral anterior rectus abdominis sheath turnover flap method for early fascial closure in patients requiring open abdominal management. World J Surg 31(1): 2-8; discussion 9-10, 2007. PMID: 17103095. DOI: $10.1007 / \mathrm{s} 00268-006-0282-3$

20 Vranckx JJ, Stoel AM, Segers K and Nanhekhan L: Dynamic reconstruction of complex abdominal wall defects with the pedicled innervated vastus lateralis and anterolateral thigh piva flap. J Plast Reconstr Aesthet Surg 68(6): 837-845, 2015. PMID: 25964228. DOI: 10.1016/j.bjps.2015.03.009

21 Lin SJ and Butler CE: Subtotal thigh flap and bioprosthetic mesh reconstruction for large, composite abdominal wall defects. Plast Reconstr Surg 125(4): 1146-1156, 2010. PMID: 20072081. DOI: 10.1097/PRS.0b013e3181d18196

22 Kuo YR, Kuo MH, Lutz BS, Huang YC, Liu YT, Wu SC, Hsieh $\mathrm{KC}$, Hsien $\mathrm{CH}$ and Jeng SF: One-stage reconstruction of large midline abdominal wall defects using a composite free anterolateral thigh flap with vascularized fascia lata. Ann Surg 239(3): 352-358, 2004. PMID: 15075651. DOI: 10.1097/01.sla.0000114229.89940.e8

23 Song YG, Chen GZ and Song YL: The free thigh flap: A new free flap concept based on the septocutaneous artery. Br J Plast Surg 37(2): 149-159, 1984. PMID: 6713155. DOI: 10.1016/00071226(84)90002-x

24 Wei FC, Jain V, Celik N, Chen HC, Chuang DC and Lin CH: Have we found an ideal soft-tissue flap? An experience with 672 anterolateral thigh flaps. Plast Reconstr Surg 109(7): 2219-2226; discussion 2227-2230, 2002. PMID: 12045540. DOI: 10.1097/00006534-200206000-00007

25 Lv Y, Cao D, Guo F, Qian Y, Wang C and Wang D: Abdominal wall reconstruction using a combination of free tensor fasciae lata and anterolateral thigh myocutaneous flap: A prospective study in 16 patients. Am J Surg 210(2): 365-373, 2015. PMID: 25824825. DOI: $10.1016 /$ j.amjsurg.2014.11.008
26 Kanters AE, Krpata DM, Blatnik JA, Novitsky YM and Rosen MJ: Modified hernia grading scale to stratify surgical site occurrence after open ventral hernia repairs. J Am Coll Surg 215(6): 787-793, 2012. PMID: 22999328. DOI: 10.1016/j.jamcollsurg.2012.08.012

27 Krpata DM, Schmotzer BJ, Flocke S, Jin J, Blatnik JA, Ermlich B, Novitsky YW and Rosen MJ: Design and initial implementation of herqles: A hernia-related quality-of-life survey to assess abdominal wall function. J Am Coll Surg 215(5): 635-642, 2012. PMID: 22867715. DOI: 10.1016/j.jamcollsurg.2012.06.412

28 di Summa PG, Matter M, Kalbermatten DF, Bauquis O and Raffoul W: Transabdominal-pelvic-perineal (tapp) anterolateral thigh flap: A new reconstructive technique for complex defects following extended abdominoperineal resection. J Plast Reconstr Aesthet Surg 69(3): 359-367, 2016. PMID: 26732292. DOI: 10.1016/j.bjps.2015.10.044

29 Medgyesi S: A successful operation for lymphoedema using a myocutaneous flap as a "wick". Br J Plast Surg 36(1): 64-66, 1983. PMID: 6821730. DOI: 10.1016/0007-1226(83)90014-0

30 Fernandez-Alvarez JA, Barrera-Pulido F, Lagares-Borrego A, Narros-Gimenez R, Gacto-Sanchez $P$ and Gomez-Cia T: Coverage of supraumbilical abdominal wall defects: The tunnelled-pedicled alt technique. Microsurgery 37(2): 119-127, 2017. PMID: 26109324. DOI: 10.1002/micr.22437

31 Zelken JA, AlDeek NF, Hsu CC, Chang NJ, Lin CH and Lin CH: Algorithmic approach to lower abdominal, perineal, and groin reconstruction using anterolateral thigh flaps. Microsurgery 36(2): 104-114, 2016. PMID: 25487137. DOI: 10.1002/micr.22354

32 Patel NG, Ratanshi I and Buchel EW: The best of abdominal wall reconstruction. Plast Reconstr Surg 141(1): 113e-136e, 2018. PMID: 29280882. DOI: 10.1097/PRS.0000000000003976

33 Scaife J, Harrison K, Romanchikova M, Parker A, Sutcliffe M, Bond S, Thomas S, Freeman S, Jena R, Bates A and Burnet N: Random variation in rectal position during radiotherapy for prostate cancer is two to three times greater than that predicted from prostate motion. Br J Radiol 87(1042): 20140343, 2014. PMID: 25138155. DOI: 10.1259/bjr.20140343

34 Kimata Y, Uchiyama K, Sekido M, Sakuraba M, Iida H, Nakatsuka $\mathrm{T}$ and Harii $\mathrm{K}$ : Anterolateral thigh flap for abdominal wall reconstruction. Plast Reconstr Surg 103(4): 1191-1197, 1999. PMID: 10088506. DOI: 10.1097/00006534-199904040-00014

35 Rosen MJ, Krpata DM, Ermlich B and Blatnik JA: A 5-year clinical experience with single-staged repairs of infected and contaminated abdominal wall defects utilizing biologic mesh. Ann Surg 257(6): 991-996, 2013. PMID: 23426340. DOI: 10.1097/SLA.0b013e3182849871

36 Nahabedian MY, Tsangaris $T$ and Momen B: Breast reconstruction with the diep flap or the muscle-sparing (ms-2) free tram flap: Is there a difference? Plast Reconstr Surg 115(2): 436-444; discussion 445-436, 2005. PMID: 15692347. DOI: 10.1097/01.prs.0000149404.57087.8e

37 Bajaj AK, Chevray PM and Chang DW: Comparison of donorsite complications and functional outcomes in free musclesparing tram flap and free diep flap breast reconstruction. Plast Reconstr Surg 117(3): 737-746; discussion 747-750, 2006. PMID: 16525258. DOI: 10.1097/01.prs.0000200062.97265.fb

Received October 14, 2019

Revised October 27, 2019

Accepted October 29, 2019 